\title{
Techniques Essay
}

\section{RNAi Applications in Mammalian Cells}

\author{
Lisa Scherer and John J. Rossi \\ Division of Molecular Biology, Beckman Research Institute of the City of Hope, Duarte, CA, USA
}

RNA interference, or RNAi, is a gene silencing mechanism originally elucidated in plants, Caenorhabditis elegans, and Drosophila $(1,2)$. Mechanistically, RNAi results in sequence-specific destruction of mRNAs, allowing targeted knockdown of gene expression. Subsequent to the discovery of RNAi in plants and lower eukaryotes, it was demonstrated that RNAi occurs in mammalian cells $(3,4)$, a finding that has prompted considerable interest in RNAi as a genetic tool and therapeutic agent. Gene silencing via the RNAi pathway appears to be activated by a double-stranded RNA (dsRNA) "trigger," which is processed by a cellular RNase III family enzyme called Dicer into short 21-25 nucleotide dsRNAs referred to as small interfering RNAs (siRNAs). The siRNAs are unwound, and one of the two strands becomes incorporated into a multiprotein complex that has been termed the RNA-induced silencing complex or RISC (5). The strand that enters RISC from the siRNA duplex appears to be the one with the less thermodynamically stable $5^{\prime}$ end $(6,7)$. Once in RISC, the siRNA strand hybridizes with complementary target mRNA(s), activating an endonuclease within RISC that cleaves the target, resulting in its destruction. RISC can subsequently be recycled and can degrade multiple target mRNAs. This article will give an overview of the methods and some examples of RNAi utilization in mammalian cells.

\section{siRNA Design: General Parameters}

Proper design is a critical consideration for siRNA-mediated silencing. Two basic siRNA schemes have been successfully employed. The first strategy uses two 21 nucleotide RNAs, typically with a twonucleotide overhang at each $3^{\prime}$ end, that mimic the natural product of Dicer cleavage (Figure 1). The second approach, referred to as short hairpin RNAs, or shRNAs (Figure 1), uses sense and antisense sequences connected by a hairpin loop. RNA duplexes slightly longer than 21 nucleotides in length can also be used as these may be further processed intracellularly into 21-25 base siRNAs. Both siRNAs and shRNAs can be chemically synthesized and introduced into cells or expressed endogenously from a promoter. For endogenous expression, RNA polymerase III (Pol III) promoters have been utilized for transcribing si- and shRNAs, since they can be engineered short hairpin RNA. to initiate transcription with the first base of the si/shRNA and because Pol III terminates transcription within a short run of $4-6$ uridines $(8,9)$. RNA polymerase II promoters have also been successfully used (10).

RNAi has also been elicited by transfecting cells with pools of siRNAs generated by cleavage of in vitro transcribed long dsRNAs with recombinant Dicer or RNase III $(11,12)$ or with Escherichia coli RNase III (13). Pooled siRNAs usually

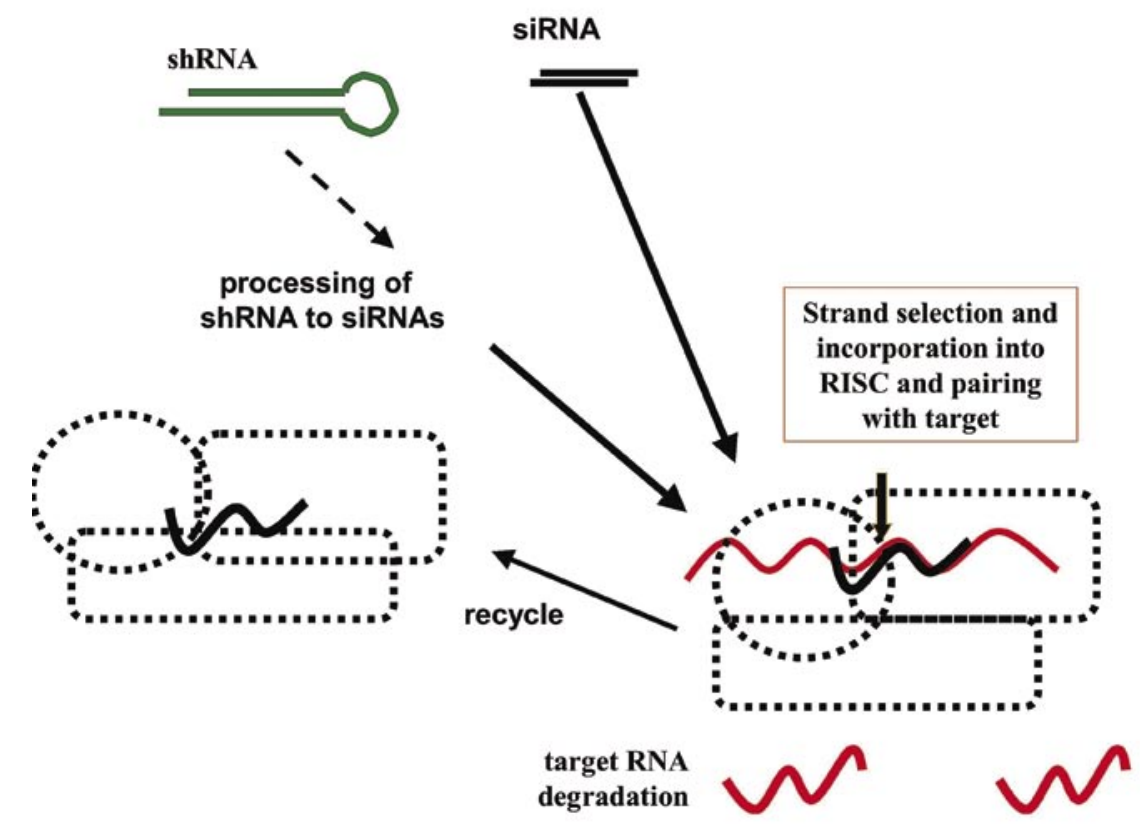

Figure 1. Mechanism of siRNA-directed degradation of target mRNA in mammalian cells. shRNAs are processed to siRNAs, which have a two base $3^{\prime}$ overhang and recessed 5' phosphate. The antisense strand of the siRNA (relative to the target) is incorporated into the RNA induced silencing complex (RISC). RISC is comprised of several proteins, at least one of which is an Argonaut family member in addition to an RNase. The siRNA directs targeted cleavage of the mRNA and the RISC complex can recycle. siRNA, small interfering RNA; shRNA,

result in potent knockdown of target mRNA(s) while allowing discrimination between homologous sequences in gene families or alternatively spliced mRNAs.

The choice of exogenously created siRNA or vector-delivered siRNA expression units will ultimately depend upon the application. Transfection of synthetic siRNAs provides only transient RNAi, but gives dose-response relationships that are easily controlled, making it more suitable for large-scale applications and multiplexing. On the other 
hand, stable, endogenous expression of si/shRNAs from integrating or episomal replicating vectors can cause longterm inhibition of gene expression-an important consideration for some therapeutic applications, such as the treatment of chronic viral infections.

\section{Selectivity of RNAi}

Some examples of siRNAs that are highly sequence-specific can be found in studies using RNAi in cancers that are associated with novel fusion transcripts. In chronic myeloid leukemia (CML), fusion bcrlab/ tran-

scripts are present in nearly all CML patients, as well as in $30 \%$ of adults with acute lymphoblastic leukemia (ALL). Transiently transfected siRNAs directed against the region spanning the bcrlabl fusion joint specifically reduced levels of the fusion bcr/ab/ transcripts and of the encoded p210 protein, without affecting the endogenous $a b /$ and $b c r$ messages, despite the fact that parts of the siRNAs share perfect complementarity with these nonfusion transcripts $(14,15)$. Importantly, the siRNA-induced reduction of the oncogenic transcript resulted in a desired physiological effect of cells becoming more susceptible to apoptosis. High selectivity will be required in therapeutic applications for which delivery to non-leukemic cells cannot be controlled.

A cautionary note about selectivity comes from a study of Pol III expressed siRNAs targeting the fusion transcript characteristic of Ewing's sarcoma (16). In this disease, the translocation $\mathrm{t}(11 ; 22)(\mathrm{q} 24 ; \mathrm{q} 12)$ produces an oncogenic EWS/Fli-1 fusion protein detected in $85 \%$ of Ewing's sarcoma and primitive neuroectodermal tumor cells. Two different expressed siRNAs targeting sequences specific to the fusion were utilized. Both EWS/Fli-1 siRNAS specifically reduced the fusion mRNA relative to cellular $\beta$-actin. However, one of the siRNAs containing 17 bases of homology to Fli-1 partially reduced endogenous Fli-1 mRNA and also resulted in unanticipated $\mathrm{N}$-myc down-regulation, highlighting the importance of monitoring cross-suppression of endogenous transcripts when using RNAi.

\section{Off-Target Effects}

There are conflicting reports about the potential "off-targeting" of siRNAs in mammalian cells. One study of siRNAs targeting a reporter construct (used in concert with DNA array analyses) showed no significant changes in the expression levels of nontargeted endogenous cellular genes (17). In contrast, other investigations have demonstrated numerous off-target effects $(18-20)$. In one case, the off-target effects were concentration-dependent, with higher concentrations of siRNAs generating more off-targeting (20). It is therefore important to iden- tify siRNA targets that produce desired effects at minimal (nanomolar) concentrations to minimize unintended effects and to design appropriate controls in siRNA knockdown experiments.

\section{Target Sequence Selection}

An siRNA's ability to stimulate degradation of its target RNA is heavily dependent on target sequence selection $(3,7,12,13,21,22)$, but the reasons are not fully understood. Base composition (GC content) alone does not seem to

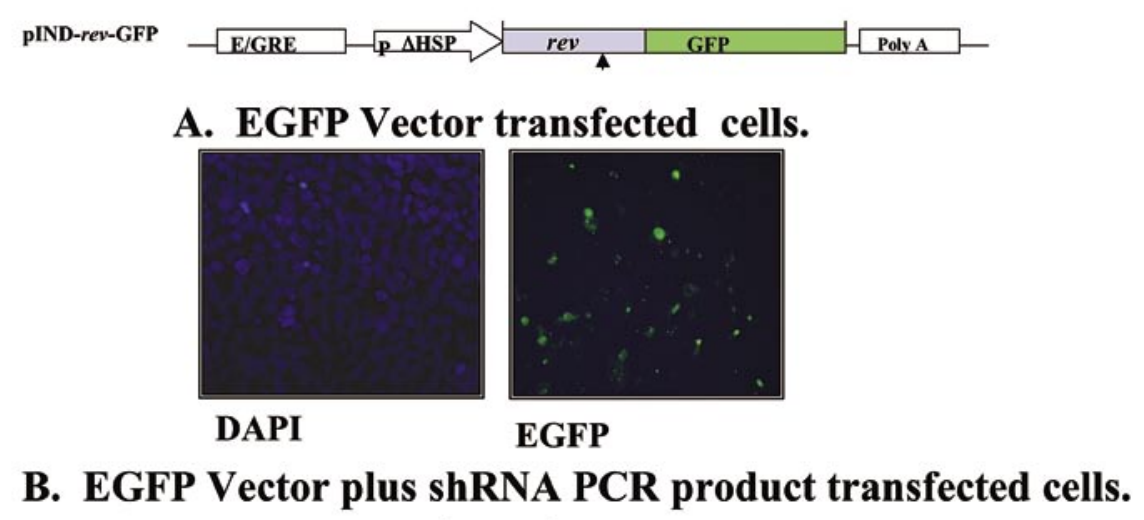

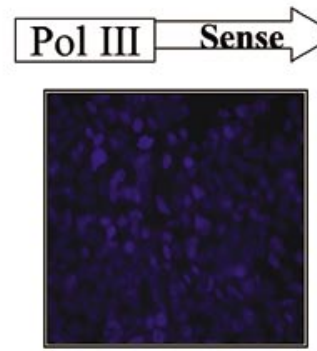

DAPI

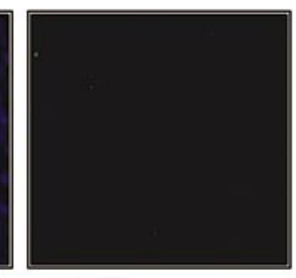

EGFP
Figure 2. Example of a target fused to a reporter gene. The HIV-1 Rev cDNA has been joined to the EGFP to monitor RNAi function of anti-Rev shRNAs. Depicted at the top of the figure is an inducible system in which the Ecdysone response elements are fused to a basal promoter, and the system is induced with the Ecdysone analogue Ponasterone $A(7,24)$. The target site for the antiRev shRNA expressed in panel B is depicted by a small vertical arrow. (A) HEK 293 cells transfected with the inducible Rev-EGFP expression vector alone. (B) HEK 293 cells transfected with Rev-EGFP fusion vector plus an anti-Rev encoding shRNA PCR product. A Pol III shRNA expression cassette is depicted. This was produced by PCR amplification as described in the text. The sense, antisense, and terminator region (Ts) are depicted. (A and B) The cell nuclei are stained with DAPI in the lefthand panels, and the right-hand panels depict EGFP fluorescence. The anti-Rev shRNA encoded by the transfected PCR product has down-regulated EGFP expression in the right-hand panel of B. HIV-1, human immunodeficiency virus type 1; EGFP, enhanced green fluorescent protein; RNAi, RNA interference; shRNA, short hairpin RNA; Pol III, RNA polymerase III. be a factor (12). More subtle structural aspects of the siRNA itself and of the target sequence, such as the ability to adopt the A-form helical structure (23) common to dsRNAs, may affect the efficiency of target site cleavage within the RISC complex. Studies utilizing methods to determine optimal in vivo ribozyme or antisense DNA target sites may be instructive. General RNA folding prediction software, such as mfold, do not reliably predict useful siRNA target sequences (see http://www.bioinfo. 
rpi.edu/ zukerm/seqanal/), but several commercial and academic web sites have useful algorithms for choosing siRNA target sequences. None of the in silico methods above takes into consideration the role of RNA chaperone proteins on inter- and intramolecular RNA interactions. A cell extract method that scans transcripts with inexpensive DNA oligonucleotides has proven to be useful for identifying ribozyme, antisense, and RNAi-susceptible target sequences $(4,18-20)$.

Another technique for identifying effective RNAi target sequences is to use a construct that includes the target gene of interest fused to a reporter, such as the enhanced green fluorescent protein (EGFP) (24) (Figure 2). The siRNAdirected cleavage of the target gene sequence will result in loss of EGFP fluorescence, which can be quantitated. This approach has several advantages: (i) in vivo application, (ii) rapid readout, and (iii) effectiveness in standard tissue culture.

In an effort to rapidly and efficiently identify RNAi sensitive sequences in cells, we developed a PCR-based procedure for generating Pol III shRNA transcription units. The in vitro-amplified PCR products are transfected into mammalian cells containing the target mRNA (24). A number of transcription units expressing different si/shRNAs targeting different sequences in the gene of interest can be rapidly synthesized and tested, since the transcriptional units are tagged with a $3^{\prime}$ sequence that allows easy PCR amplification from transfected cells, enabling researchers to test multiple shRNA sequences and isolate the most potent ones following cell sorting (24).

\section{Targeting Human Immunodeficiency Virus with RNAi}

Human immunodeficiency virus (HIV) has become a prime target for RNAi due to problems with resistance to current drugs and the high cost of new drug development $(25,26)$. Synthetic and expressed siRNAs have been used to target a number of early and late viral mRNAs as well as cellular cofactors $(7,27,28)$. Successful inhibition of HIV replication has been achieved in numerous human cell lines and primary cells including hematopoietic stem cell-derived T lymphocytes and macrophages (29).

The use of siRNA as a potential therapeutic agent in the treatment of HIV and hepatitis C virus (HCV) (30-33) is complicated by the high mutation rates of these viruses during replication. Since even one nucleotide mismatch between an siRNA and its target can drastically lower activity $(6,34)$, the appearance of escape mutants is likely. Selecting targets in highly conserved sequences (where mutations are presumably deleterious to the virus) and using combinations of si/shRNAs targeting different sites within the HIV viral genome will reduce the likelihood of resistant variants arising. The multitargeting approaches of RNAi make this method a potentially powerful therapeutic for the treatment of viral infection.

\section{Conclusion}

RNAi has rapidly gained in popularity as the method of choice for target-specific down-regulation of gene expression in eukaryotic systems. Because unwanted side effects can occur, careful attention to controls must be utilized in RNAi-mediated knockdowns. As we learn more about the mechanisms involved in this phenomenon, it may be possible to increase specificity and avoid off-targeting problems by proper design of si/shRNAs. It is clear that use of this powerful biological phenomenon will continue to expand and provide fascinating new insights into gene regulation as well as exciting therapeutic applications.

\section{References}

1.Hannon, G.J. 2002. RNA interference. Nature 418:244-251.

2.Bernstein, E., A.M. Denli, and G.J. Hannon. 2001. The rest is silence. RNA 7:1509-1521

3.Elbashir, S.M., J. Harborth, W. Lendeckel, A. Yalcin, K. Weber, and T. Tuschl. 2001. Duplexes of 21-nucleotide RNAs mediate RNA interference in cultured mammalian cells. Nature 411:494-498.

4.Caplen, N.J., S. Parrish, F. Imani, A. Fire, and R.A. Morgan. 2001. Specific inhibition of gene expression by small double-stranded RNAs in invertebrate and vertebrate systems. Proc. Natl. Acad. Sci. USA 98:9742-9747.

5.Agrawal, N., P.V. Dasaradhi, A. Mohmmed, P. Malhotra, R.K. Bhatnagar, and S.K. Mukherjee. 2003. RNA interference: biology, mechanism, and applications. Microbiol. Mol. Biol. Rev. 67:657-685.

6.Paul, C.P., P.D. Good, I. Winer, and D.R. Engelke. 2002. Effective expression of small interfering RNA in human cells. Nat. Biotechnol. 20:505-508.

7.Lee, N.S., T. Dohjima, G. Bauer, H. Li, M.J. Li, A. Ehsani, P. Salvaterra, and J. Rossi. 2002. Expression of small interfering RNAs targeted against HIV-1 rev transcripts in human cells. Nat. Biotechnol. 20:500-505.

8.Khvorova, A., A. Reynolds, and S.D. Jayasena. 2003. Functional siRNAs and miRNAs exhibit strand bias. Cell 115:209-216.

9.Schwarz, D.S., G. Hutvagner, T. Du, Z. Xu, N. Aronin, and P.D. Zamore. 2003. Asymmetry in the assembly of the RNAi enzyme complex. Cell 115:199-208

10.Xia, H., Q. Mao, H.L. Paulson, and B.L. Davidson. 2002. siRNAmediated gene silencing in vitro and in vivo. Nat. Biotechnol. 20:10061010.

11.Myers, J.W., J.T. Jones, T. Meyer, and J.E. Ferrell. 2003. Recombinant Dicer efficiently converts large dsRNAs into siRNAs suitable for gene silencing. Nat. Biotechnol. 21:324-328

12.Kawasaki, H., E. Suyama, M. Iyo, and K. Taira. 2003. siRNAs generated by recombinant human Dicer induce specific and significant but target site-independent gene silencing in human cells. Nucleic Acids Res. 31:981-987.

13.Yang, D., F. Buchholz, Z. Huang, A. Goga, C.Y. Chen, F.M. Brodsky, and J.M. Bishop. 2002. Short RNA duplexes produced by hydrolysis with Escherichia coli RNase III mediate effective RNA interference in mammalian cells. Proc. Natl. Acad. Sci. USA 99:9942-9947.

14.Wilda, M., U. Fuchs, W. Wossmann, and A. Borkhardt. 2002. Killing of leukemic cells with a BCR/ABL fusion gene by RNA interference (RNAi). Oncogene 21:5716-5724

15.Scherr, M., K. Battmer, T. Winkler, O. Heidenreich, A. Ganser, and $\mathbf{M}$. Eder. 2003. Specific inhibition of bcr-abl gene expression by small interfering RNA. Blood 101:1566-1569.

16.Dohjima, T., N.S. Lee, H. Li, T. Ohno, and J.J. Rossi. 2003. Small interfering RNAs expressed from a Pol III promoter suppress the EWS/ Fli-1 transcript in an Ewing sarcoma cell line. Mol. Ther. 7:811-816.

17.Chi, J.T., H.Y. Chang, N.N. Wang, D.S. Chang, N. Dunphy, and P.O. Brown. 2003. Genomewide view of gene silencing by small interfering RNAs. Proc. Natl. Acad. Sci. USA 100:6343-6346.

18.Saxena, S., Z.O. Jonsson, and A. Dutta. 2003. Small RNAs with imperfect match to endogenous mRNA repress translation. Implications for off-target activity of small inhibitory RNA in mammalian cells. J. Biol. Chem. 278:44312-44319.

19. Jackson, A.L., S.R. Bartz, J. Schelter, S.V. Kobayashi, J. Burchard, M. Mao, B. Li, G. Cavet, and P.S. Linsley. 2003. Expression profiling reveals off-target gene regulation by RNAi. Nat. Biotechnol. 21:635637. 


\section{Techniques Essay}

20.Persengiev, S.P., X. Zhu, and M.R. Green. 2004. Nonspecific, concentration-dependent stimulation and repression of mammalian gene expression by small interfering RNAs (siRNAs). RNA 10:12-18.

21.Holen, T., M. Amarzguioui, M.T. Wiiger, E. Babaie, and H. Prydz. 2002. Positional effects of short interfering RNAs targeting the human coagulation trigger Tissue Factor. Nucleic Acids Res. 30:1757-1766.

22.Yu, J.Y., S.L. DeRuiter, and D.L. Turner. 2002. RNA interference by expression of short-interfering RNAs and hairpin RNAs in mammalian cells. Proc. Natl. Acad. Sci. USA 99:6047-6052.

23.Chiu, Y.L. and T.M. Rana. 2002. RNAi in human cells: basic structural and functional features of small interfering RNA. Mol. Cell 10:549-561.

24.Castanotto, D., H. Li, and J.J. Rossi. 2002. Functional siRNA expression from transfected PCR products. RNA 8:1454-1460.

25.Martinez, M.A., B. Clotet, and J.A. Este. 2002. RNA interference of HIV replication. Trends Immunol. 23:559-561.

26.Kitabwalla, M. and R.M. Ruprecht. 2002. RNA interference-a new weapon against HIV and beyond. N. Engl. J. Med. 347:1364-1367.

27.Coburn, G.A. and B.R. Cullen. 2002. Potent and specific inhibition of human immunodeficiency virus type 1 replication by RNA interference. J. Virol. 76:9225-9231.

28.Surabhi, R.M. and R.B. Gaynor. 2002. RNA interference directed against viral and cellular targets inhibits human immunodeficiency Virus
Type 1 replication. J. Virol. 76:12963-12973.

29.Banerjea, A., M.-J. Li, L. Remling, N.-S. Lee, J. Rossi, and R. Akkina. 2003. Potent inhibition of HIV-1 by lentiviral vector transduced siRNAs in primary humanT-lymphocytes differentiated in SCID-hu mice and CD34+ progenitor cell derived macrophages. Mol. Ther. 8:62-71.

30.Tang, H., T. Peng, and F. Wong-Staal. 2002. Novel technologies for studying virus-host interaction and discovering new drug targets for HCV and HIV. Curr. Opin. Pharmacol. 2:541-547.

31.Yokota, T., N. Sakamoto, N. Enomoto, Y. Tanabe, M. Miyagishi, S. Maekawa, L. Yi, M. Kurosaki, et al. 2003. Inhibition of intracellular hepatitis $C$ virus replication by synthetic and vector-derived small interfering RNAs. EMBO Rep. 4:602-608.

32.Kapadia, S.B., A. Brideau-Andersen, and F.V. Chisari. 2003. Interference of hepatitis $C$ virus RNA replication by short interfering RNAs. Proc. Natl. Acad. Sci. USA 100:2014-2018.

33.Seo, M.Y., S. Abrignani, M. Houghton, and J.H. Han. 2003. Small interfering RNA-mediated inhibition of hepatitis $C$ virus replication in the human hepatoma cell line Huh-7. J. Virol. 77:810-812.

34.Jacque, J.M., K. Triques, and M. Stevenson. 2002. Modulation of HIV-1 replication by RNA interference. Nature 418:435-438. 